Article

\title{
A Thermal Model for Carbon Nanotube Interconnects
}

\section{Kaji Muhammad Mohsin ${ }^{1}$, Ashok Srivastava ${ }^{1, *}$, Ashwani K. Sharma ${ }^{2}$ and Clay Mayberry ${ }^{2}$}

1 School of Electrical Engineering and Computer Science, Louisiana State University, Baton Rouge, LA 70803, USA; E-Mail: kmohsi1@1su.edu

2 Air Force Research Laboratory/Space Electronics Branch, Space Vehicles Directorate, Electronics Foundations Group, 3550 Aberdeen Avenue SE, Kirtland, NM 87117, USA; E-Mails: ashwani.sharma@kirtland.af.mil (A.K.S.); clay.mayberry@kirtland.af.mil (C.M.)

* Author to whom correspondence should be addressed; E-Mail: ashok@ece.lsu.edu; Tel.: +1-225-578-5622; Fax: +1-225-578-5200.

Received: 11 March 2013; in revised form: 10 April 2013 / Accepted: 12 April 2013 / Published: 26 April 2013

\begin{abstract}
In this work, we have studied Joule heating in carbon nanotube based very large scale integration (VLSI) interconnects and incorporated Joule heating influenced scattering in our previously developed current transport model. The theoretical model explains breakdown in carbon nanotube resistance which limits the current density. We have also studied scattering parameters of carbon nanotube (CNT) interconnects and compared with the earlier work. For $1 \mu \mathrm{m}$ length single-wall carbon nanotube, $3 \mathrm{~dB}$ frequency in $\mathrm{S}_{12}$ parameter reduces to $\sim 120 \mathrm{GHz}$ from $1 \mathrm{THz}$ considering Joule heating. It has been found that bias voltage has little effect on scattering parameters, while length has very strong effect on scattering parameters.
\end{abstract}

Keywords: SWCNT; VLSI interconnect; Joule heating; scattering

\section{Introduction}

The current complementary metal oxide semiconductor (CMOS) technology in nm- and sub-nm node for very large scale integration (VLSI) is facing challenges due to performance limitation of $\mathrm{Cu} /$ low-k dielectric material as an interconnection, because of increased resistivity of $\mathrm{Cu}$, electromigartion and void formation [1,2]. Many alternatives to $\mathrm{Cu}$ interconnection in nanometer technology node have been proposed in the literature, including optical interconnects. The $1 \mathrm{D}$ carbon nanotubes and 2D graphene nanoribbons are found to be very promising alternatives to current $\mathrm{Cu}$ 
interconnects for use in nm-CMOS technology [3]. Early discovery of carbon nanotubes in 1991 by Iijima [4] and its excellent electrical, mechanical and thermal properties and established synthesis techniques [5-9], have led to major R\&D efforts in integrating carbon nanotubes in CMOS processes [6-10], whereas graphene nanoribbon as a possible substitute for $\mathrm{Cu}$ interconnect is also evolving [11].

Though carbon nanotube has high thermal conductivity, it has been observed experimentally that the conducting carbon nanotube breaks down due to Joule heating which thus limits its current density [11,12]. Notable work has been done in electrical modeling of carbon nanotube as an interconnect material, substituting $\mathrm{Cu} / \mathrm{low}-\mathrm{k}$ from quantum and classical considerations [13-15]. Recently, Srivastava et al. [16] have conducted exhaustive studies on single-wall carbon nanotube interconnect from one-dimensional fluid model and included electron-electron repulsive interaction and extended to multi-wall carbon nanotubes and bundles of single-wall carbon nanotubes [17]. Thermal effects in VLSI interconnect limits the current density and there have been problems of breakdown in carbon nanotubes due to resistive heating [18-21]. Huang et al. [22] have studied thermal transport and observed experimentally that the hottest spot is located at the center of the tube from where breakdown is initiated. The current transport is diffusive and not ballistic. Santini [23] has done exhaustive experimental studies on Joule heating-induced breakdown of carbon nanotube interconnects and attribute to the defect sites as also observed by Huang et al. [22]. Naeemi and Meindl [20,21] have studied temperature coefficient of resistance of single- and multi-wall carbon nanotube interconnects and related to various electron-phonon scattering mechanisms. Kitsuki et al. [24] and Yamada et al. [25] have explained experimental results of current induced breakdown in carbon nano fibers. In this work, we have examined the problem of Joule heating in carbon nanotube interconnects based on one-dimensional fluid model of electronic transport considering various scattering mechanisms and studied the temperature distribution across the length of the nanotube and scattering parameters. In Section 2 of the paper, we have developed one-dimensional fluid model incorporating thermal effects. In Section 3, temperature profiling across the length of the carbon nanotube is presented followed by the results and discussion. Conclusions are presented in Section 4.

\section{Electrical and Thermal Transport Model}

The conducting single-wall, multi-wall and bundle of single-wall carbon nanotube have been considered for possible replacement of $\mathrm{Cu} / \mathrm{low}-\mathrm{k}$ dielectric interconnects used in current CMOS technology depending upon the type of the interconnect, such as whether local or global. In the present work, we have considered Joule heating in a metallic single-wall carbon nanotube for better understanding of breakdown and also for analytical simplicity.

In our earlier work [16], we have made modification in two-dimensional fluid model and included electron-electron repulsive interaction and considered distribution of conduction electrons on the lateral surface of single-wall carbon nanotube (SWCNT) cylindrical shell. The analysis reduces to semi-classical one-dimensional fluid model. The SWCNT is a two-dimensional graphene sheet rolled to form a cylindrical nanotube of infinitesimally thin layer. Thus, conduction electrons are distributed on the lateral surface of the SWCNT and motion of electrons is confined to the surface. As the diameter of CNT is very small we can also assume that electron is confined in one-dimensional space. 
We can assume that cloud of electrons is moving across the surface of the nanotube. Figure 1 shows a structure of a single wall carbon nanotube.

Figure 1. Single wall carbon nanotube.

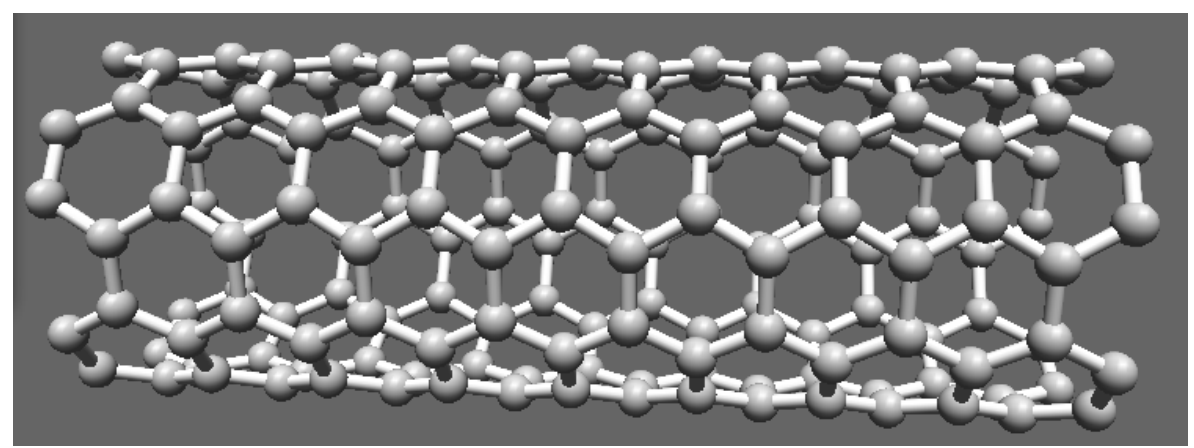

The motion of electrons across the SWCNT in a 1D fluid model can be described by the following equation $[16,17]$,

$$
\frac{\partial j(z, t)}{\partial t}+v j+u_{e}^{2} \frac{\partial \sigma(z, t)}{\partial z}=\frac{e^{2} n_{0}}{m}(1-\alpha) \varepsilon_{z}
$$

where $j$ is the current density, $m$ is effective mass of an electron, $n_{0}$ is equilibrium three-dimensional electron density and $\sigma$ is conductivity. Electron-electron repulsion factor is described by $\alpha$. The thermodynamic speed of sound is $u_{\mathrm{e}} ; e$ is electronic charge, $\varepsilon_{\mathrm{z}}$ is electric field and $v$ is relaxation frequency of electron. The Equation (1) describes the relation between an electric field and the current density.

We have modified the relaxation frequency $(v)$ in Equation (1) considering the effective mean free path as follows,

$$
v=\frac{v_{F}}{\lambda_{e f f}}
$$

In Equation (2), $v_{\mathrm{F}}$ is Fermi velocity of electron and $\lambda_{\text {eff }}$ is effective mean free path of an electron which accounts for acoustic and optical phonon scattering mechanism responsible for increased resistance of the interconnect. The spontaneous scattering length for emitting an optical phonon $\left(\lambda_{o p}\right)$ and acoustic phonon $\left(\lambda_{a c}\right)$ can be estimated as follows [26],

$$
\begin{gathered}
\lambda_{\text {op }}=56.4 \mathrm{D} \\
\lambda_{a c}=\frac{400.46^{*} 10^{3} \mathrm{D}}{T}
\end{gathered}
$$

These two scattering lengths depend on diameter of the carbon nanotube $(D)$ and surrounding temperature $(T)$. The scattering length due to optical phonon absorption, $\lambda_{o p, a b s}$ has been modeled by the following equation [19],

$$
\lambda_{o p, a b s}=\lambda_{o p} \frac{N_{o p}(300)+1}{N_{o p}(T)}
$$


In Equation (5), $N_{o p}$ describes the optical phonon occupation which can be calculated from Bose-Einstein statistics given by,

$$
N_{o p}=\frac{1}{\exp \left(\frac{\hbar \omega_{o p}}{K_{B} T}\right)-1}
$$

where $\hbar \omega_{o p}$ is optical phonon energy and its typical value varies from $0.16 \mathrm{eV}$ to $0.20 \mathrm{eV}$ [19]. In our work, we have taken this value as $0.16 \mathrm{eV}$. Occupancy function of optical phonon increases as temperature increases which eventually decreases optical phonon absorption component $\left(\lambda_{o p, a b s}\right)$ of electron mean free path. Optical phonon emission process has two components, one is for the absorbed energy $\left(\lambda_{o p, e m s}^{a b s}\right)$ and another $\left(\lambda_{o p, e m s}^{\text {fld }}\right)$ is for the electric field across the SWCNT length. Both of these components are expressed as follows [19],

$$
\begin{gathered}
\lambda_{o p, e m s}^{a b s}=\lambda_{o p, a b s}+\frac{N_{o p}(300)+1}{N_{o p}(T)+1} \lambda_{o p} \\
\lambda_{o p, e m s}^{f l d}=\frac{\hbar \omega_{o p}-K_{B} T}{q \frac{V}{l}}+\frac{N_{o p}(300)+1}{N_{o p}(T)+1} \lambda_{o p}
\end{gathered}
$$

In Equations (5), (7) and (8), $N_{o p}(300)$ is the value of phonon occupation function at $300 \mathrm{~K} . K_{\mathrm{B}}$ in Equation (8) is the Boltzmann constant. Carrier scattering path due to optical emission under the influence of electric field ( $\lambda_{o p, e m s}^{\text {fld }}$ ) depends on both the electric field $(V / l)$ and temperature $(T)$. The effective mean free path can now be calculated by Matthiessen's rule as follows,

$$
\begin{gathered}
\frac{1}{\lambda_{o p, e m s}}=\frac{1}{\lambda_{o p, e m s}^{a b s}}+\frac{1}{\lambda_{o p, e m s}^{f l d}} \\
\frac{1}{\lambda_{e f f}}=\frac{1}{\lambda_{a c}}+\frac{1}{\lambda_{o p, e m s}}+\frac{1}{\lambda_{o p, a b s}}
\end{gathered}
$$

Assuming SWCNT as a good conductor and considering only one-dimensional flow we can deduce the current density from Equation (1) which is given by,

$$
\hat{j}=\frac{e^{2} n_{0}}{m}(1-\alpha) \frac{1}{\frac{v_{F}}{\lambda_{e f f}}+i \omega} \bar{\varepsilon}
$$

where $\omega$ is the angular frequency. The current density equation relating the frequency dependence conductivity of the SWCNTs, $\sigma(\omega)$ is expressed by,

$$
\sigma(\omega)=\frac{\hat{j}}{\bar{\varepsilon}}=\frac{e^{2} n_{0}}{m}(1-\alpha) \frac{1}{\frac{v_{F}}{\lambda_{e f f}}+i \omega}
$$

$\sigma(\omega)$ is also called as dynamic conductivity of SWCNT. For metallic CNT, semi-classical axial conductivity is given by [27], 


$$
\tilde{\sigma}_{z z}=\frac{2 e^{2} v_{F}}{\pi^{2} \hbar r} \frac{1}{v+i \omega}
$$

Combining Equations (12) and (13), we obtain,

$$
\frac{n_{o}}{m}=\frac{2 v_{F}}{\pi^{2} \hbar r} \frac{1}{1-\alpha}
$$

where $r$ is the radius of SWCNT. Equation (14) relates dynamic conductivity with the semi-classical conductivity. We have assumed that SWCNT is metallic and placed above a perfectly conducting plane and also assumed that quasi-TEM electromagnetic wave is propagating through the SWCNT. The electric field can be expressed as [16],

$$
\varepsilon_{z}=R I+L_{K} \frac{\partial I}{\partial t}+\frac{1}{C_{Q}} \frac{\partial q}{\partial z}
$$

where $R$ is the resistance in per unit length, $L_{\mathrm{K}}$ is kinetic inductance in per unit length and $C_{\mathrm{Q}}$ is quantum capacitance in per unit length. The SWCNT interconnect can then be better explained as a transmission line shown in Figure 2 where its parameters are described by following equations,

$$
\begin{gathered}
L_{k}=\frac{\pi \hbar}{4 e^{2} v_{F}} \\
C_{Q}=\frac{1}{L_{K} u_{e}^{2}} \\
C_{E}=\frac{2 \pi \varepsilon}{\ln \frac{h}{2 r}} \\
R=f\left(\lambda_{\text {eff }}\right)=\frac{R_{o}}{4} \frac{1}{\lambda_{\text {eff }}} \\
L_{M}=\frac{\mu}{2 \pi} \ln \left(\frac{h}{2 r}\right)
\end{gathered}
$$

In Equation (18), $h$ is the height of SWCNT over the perfectly conducting plane and $R_{0}$ is quantum resistance taken as $12.906 \mathrm{k} \Omega$ and $L_{\mathrm{M}}$ is described by the Equation (20) as the magnetic inductance.

In thermal modeling of SWCNT interconnect material, $R(V, D)$ is the voltage $(V)$ and diameter $(D)$ dependent critical parameter which directly depends on the effective mean free path $\left(\lambda_{\text {eff }}\right)$. Increase in temperature of interconnect triggers various scattering processes which result in increase of the resistance. This increased resistance limits the current density and eventually limits heat generation. Consequently, an iterative solution is needed to find out the actual converged value for resistance instead of using a direct equation. 
Figure 2. Transmission line model of SWCNT interconnect.

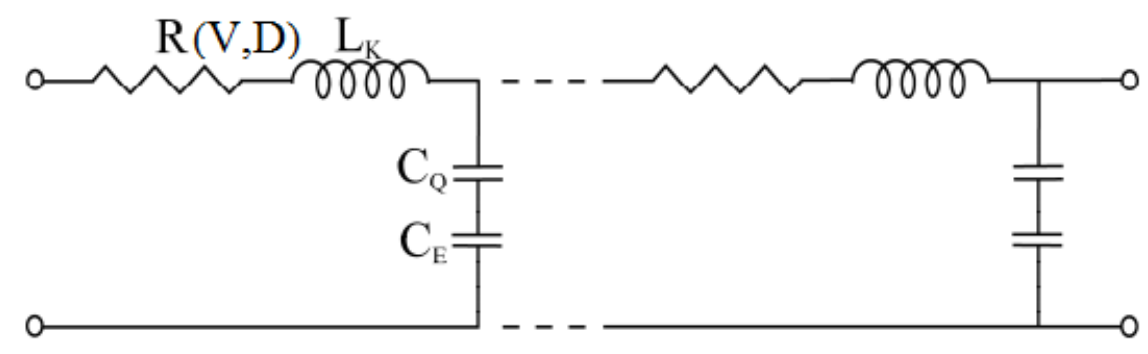

\section{Results and Discussion}

Temperature profiles along the SWCNT length can be numerically solved from steady state heat equation given by Carslaw and Jaeger [28],

$$
A \nabla(\kappa \nabla T)+p-g\left(T-T^{\prime}\right)=0
$$

where $\kappa$ is the heat conductivity, $A$ is the Cross sectional area $(\pi \times$ Diameter $\times$ Thickness $), T^{\prime}$ is ambient temperature, $g$ is thermal conductivity of the substrate and $p$ is heating source power. In calculation, the terminal temperatures of SWCNT are assumed to be at $350 \mathrm{~K}$ which is the normal operating temperature of a typical bulk semiconductor. Therefore, in Equation (21), $T^{\prime}=350 \mathrm{~K}$ and $g=0.15 \mathrm{~W} \mathrm{~m}^{-1} \mathrm{~K}^{-1}$, have been used [19]. The standard feature size of the CNT has been used so that results can be compared with the findings of other reported work. CNT diameter, D has been taken as $1 \mathrm{~nm}$ which is a typical value. Resistance of SWCNT at different biasing voltages and lengths has been calculated by numerically solving Equation (21). Since, thermal conductivity $(\kappa)$ is almost constant within $350 \mathrm{~K}$ to $800 \mathrm{~K}$ [19], we have taken thermal conductivity constant at a particular bias voltage. The steps in calculation of resistance are described as follows assuming ambient temperature remains constant as that of the CNT across its length.

1. Consider differential length of CNT and calculate mean free path as well as differential resistance for differential element using Equations (10) and (19). Estimate total resistance of SWCNT by summing all differential resistances.

2. Calculate current from Equation $I=V /\left(R+R_{\mathrm{c}}\right)$, where $R_{\mathrm{c}}$ is the contact resistance $30 \mathrm{~K} \Omega$ [19].

3. Calculate $I^{2} R$ per unit length for heat generation and then use Equation (21) to get temperature profile over the CNT length.

4. Use current temperature profile as the initial temperature for next iteration. Repeat steps 1 to 4 until convergence is obtained.

Figure 3 shows the temperature profile over the length of SWCNT of $2 \mu \mathrm{m}$ length. At different bias voltages, the temperature profile varies over the length. At biasing voltage above $3 \mathrm{~V}$, the temperature reaches close to the breakdown temperature of $873 \mathrm{~K}$ [19]. From Figure 3, it can be inferred that biasing voltage less than $4 \mathrm{~V}$ is not sufficient enough for causing the breakdown of SWCNT. It is to be mentioned that for low biasing, the iteration requires less than 5 iterations to converge. On the other hand, high bias voltage more than $4 \mathrm{~V}$, calculation takes more iteration to converge.

Figure 4 shows power dissipation versus bias voltage. For $4 \mathrm{~V}$ biasing voltage, Joule heating power is close to $0.13 \mathrm{~mW}$. As a matter of fact, at a certain bias voltage enough heat will be produced that 
will be sufficient for inducing breakdown in SWCNT at the mid-point. Higher biasing voltage increases optical phonon emission induced by the higher electric field. According to the Equation (8), electron scattering length $\lambda_{o p, e m s}^{\text {fld }}$ decreases with increase in bias voltage which contributes to decrease in effective mean free path $\left(\lambda_{e f f}\right)$. The total resistance increases with decreasing effective scattering length. This increase in resistance contributes to increase in Joule heating.

Figure 3. Temperature profile of SWCNT of $2 \mu \mathrm{m}$ length.

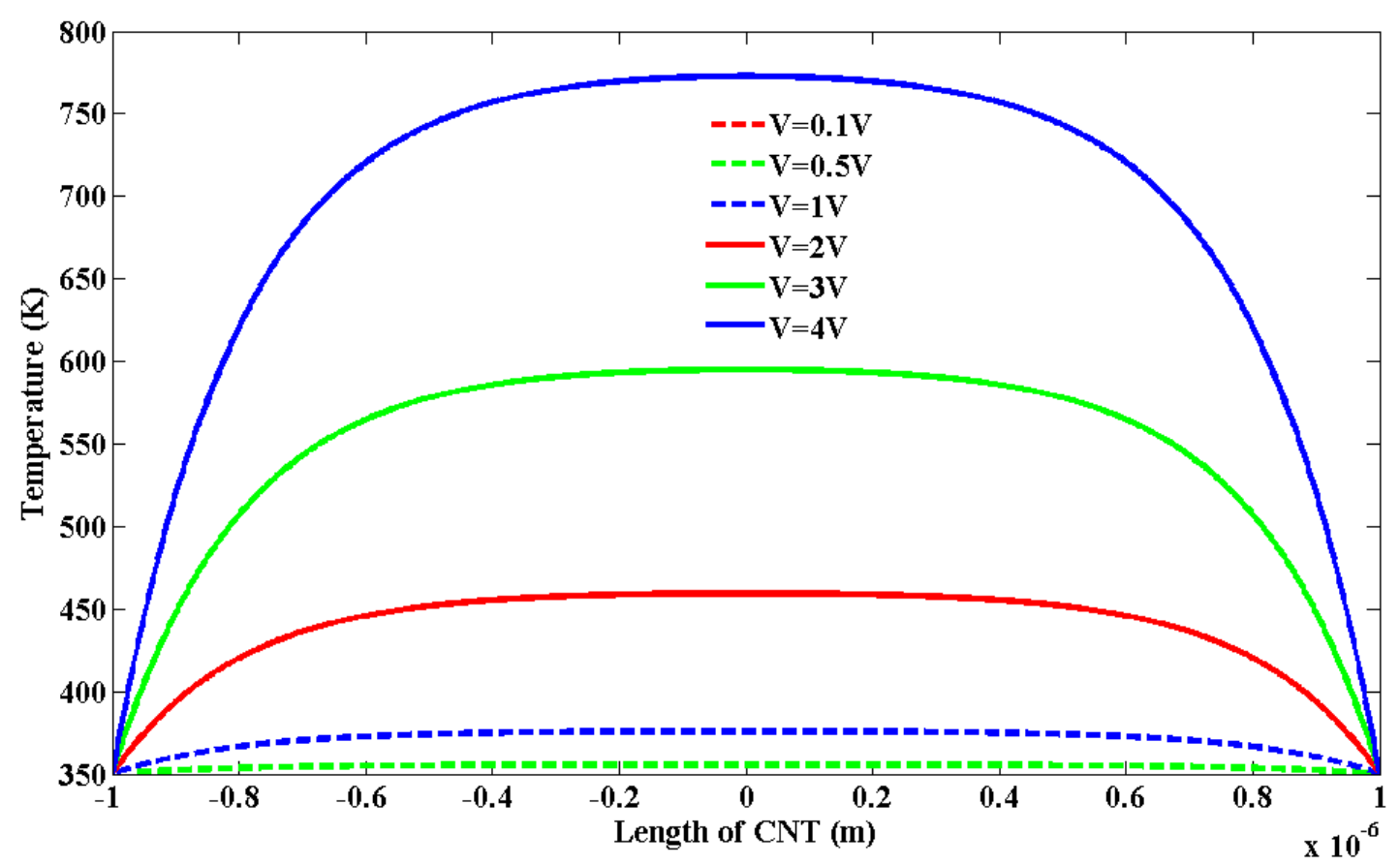

Figure 4. Power dissipation due to Joule heating along the SWCNT length.

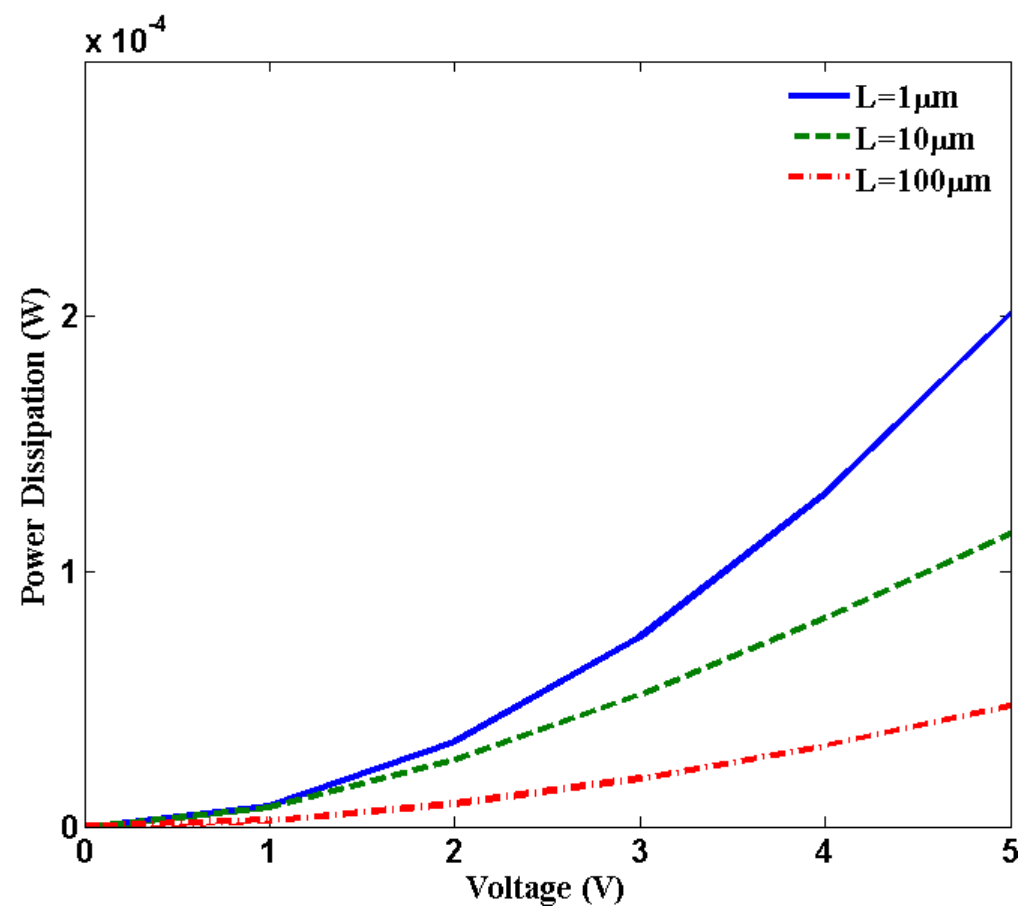


Using Equations (16)-(18), we have calculated kinetic inductance, $L_{\mathrm{K}}=3.6 \mathrm{nH} / \mu \mathrm{m}$, quantum capacitance, $C_{\mathrm{Q}}=90 \mathrm{aF} / \mu \mathrm{m}$ and electrostatic capacitance $C_{\mathrm{E}}=70 \mathrm{aF} / \mu \mathrm{m}$ of SWCNT transmission line to study S-parameters. Typically, a SWCNT diameter is $\sim 1 \mathrm{~nm}$ and oxide thickness over which SWCNT is deposited is $\sim 100 \AA$. The calculated value of $L_{\mathrm{M}} \sim 1 \mathrm{pH} / \mu \mathrm{m}$ which is very small compared to the value of $L_{\mathrm{K}}$. The calculated kinetic inductance is consistent with the value calculated in [29] for metallic CNTs. It is apparent from Equations (16)-(20) that inductance and capacitance are constant for a specific SWCNT with a given length and diameter. On the other hand, resistance of SWCNT interconnect is a function of bias voltage, SWCNT diameter, length, and temperature which influences scattering parameters. Scattering Parameter $S_{11}$ is the ratio of power reflected from the transmission line to the incident power. Scattering Parameter $S_{12}$ is the ratio of power transmitted through the transmission line to the incident power. Two port network parameters $S_{11}$ and $S_{12}$ have been calculated considering lumped elements and normalized by $50 \mathrm{ohm}$ impedance. Although transmission line is a distributed device, we have used lumped element model to calculate S-parameters for the sake of efficient computation. Figures 5 and 6 show plots of $S_{11}$ and $S_{12}$ parameters of SWCNT at $0.1 \mathrm{~V}$ bias voltage. At higher frequencies inductive and capacitive terms dominate over the resistive term which results in an oscillatory behavior of S-parameters as observed in Figures 5 and 6. Figure 5 for the $S_{11}$ parameter exhibits an oscillatory behavior for $100 \mu \mathrm{m}$ long interconnect above $10 \mathrm{GHz}$ in the frequency range studied. Figure 6 for the $S_{12}$ parameter shows an oscillatory behavior for both $10 \mu \mathrm{m}$ and $100 \mu \mathrm{m}$ long interconnects above $70 \mathrm{GHz}$ and $7 \mathrm{GHz}$, respectively. However, the short interconnect of $1 \mu \mathrm{m}$ length does not show any oscillatory behavior for $\mathrm{S}_{11}$ and $\mathrm{S}_{12}$ parameters.

Figure 5. Plot of $\mathrm{S}_{11}$ parameter of SWCNT interconnects at $0.1 \mathrm{~V}$ bias voltage.

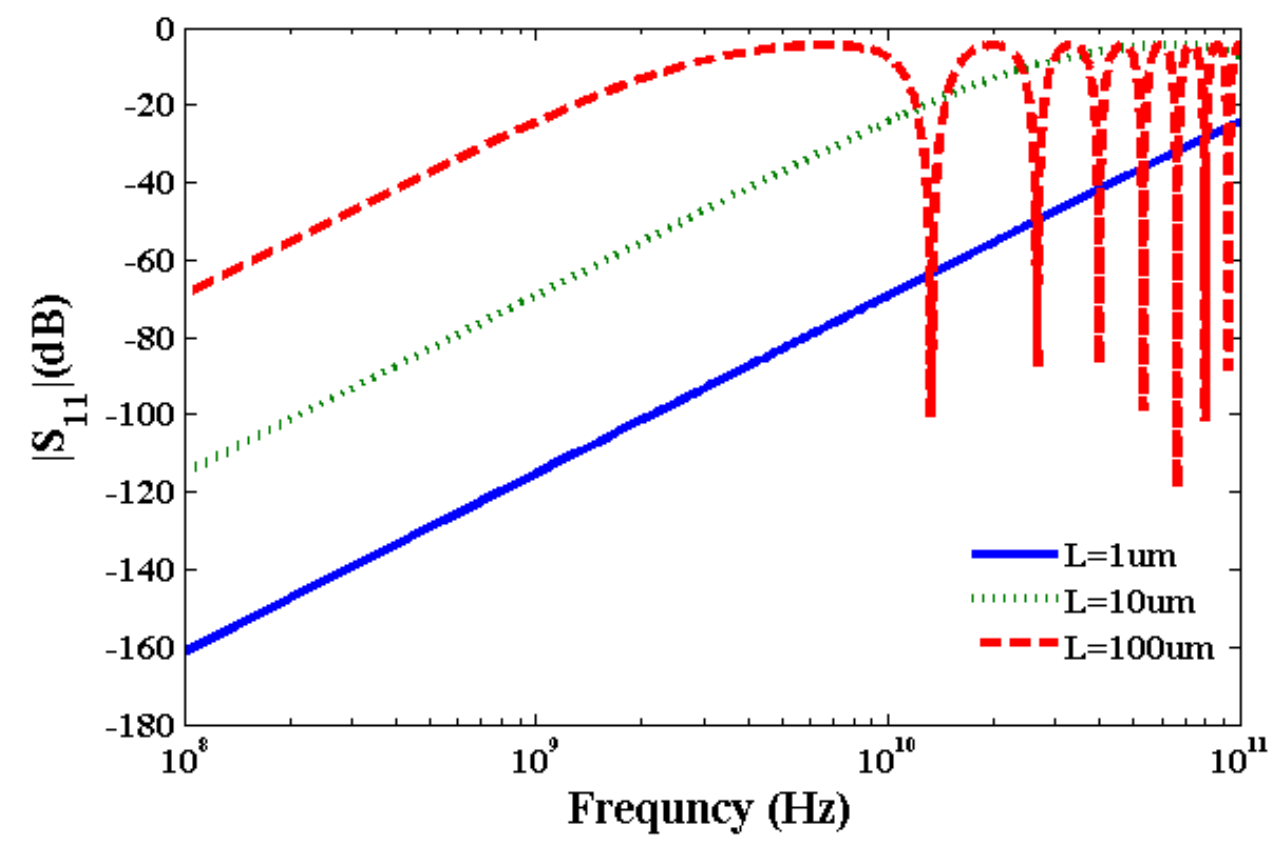


Figure 6. Plot of $\mathrm{S}_{12}$ parameter of SWCNT interconnects at $0.1 \mathrm{~V}$ bias voltage.

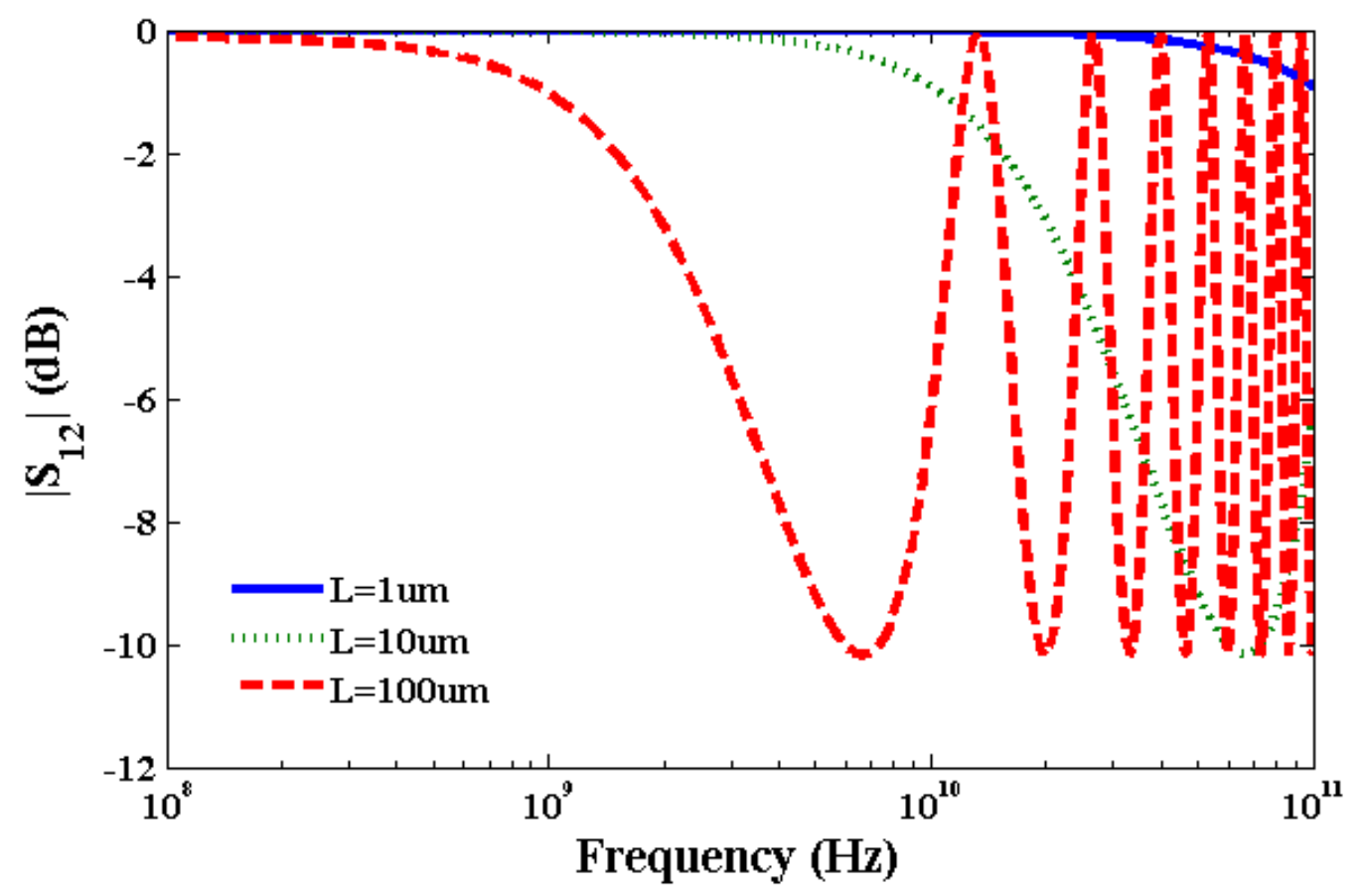

Table 1 summarizes the comparison of results with that reported in [16] without considering the Joule heating and with the Joule heating induced scattering with this current model. We have considered the frequency band in which value of $\mathrm{S}_{12}$ parameter is within $-3 \mathrm{~dB}$ of its maximum value at $0.1 \mathrm{~V}$. It is noticeable from Table 1 that the bandwidth reduces considering Joule heating due to scattering in comparison to the bandwidth without Joule heating. The frequency band width for a interconnect decreases considering Joule heating induced scattering and increased resistance.

Table 1. $\mathrm{S}_{12}$ parameters of SWCNT.

\begin{tabular}{ccc}
\hline Length of SWCNT $(\boldsymbol{\mu m})$ & $\begin{array}{c}\text { Band Width (GHz) without } \\
\text { Scattering [16] }\end{array}$ & $\begin{array}{c}\text { Band Width (GHz) with } \\
\text { Scattering }\end{array}$ \\
\hline 1 & 1000 & 120 \\
10 & 110 & 11 \\
100 & 30 & 1.0 \\
\hline
\end{tabular}

Figure 7 shows resistance variation with the applied current for short local and global interconnects lengths. It is observed that breakdown in SWCNT occurs due to Joule heating which results in an infinite increase in resistance. This theoretical observation agrees with the experimental observation [30]. Figure 8 shows the dependence of $\mathrm{S}_{12}$ on bias voltages and shows an increase in $\mathrm{S}_{12}$ with increased bias voltage. 
Figure 7. Plot of SWCNT resistance versus current.

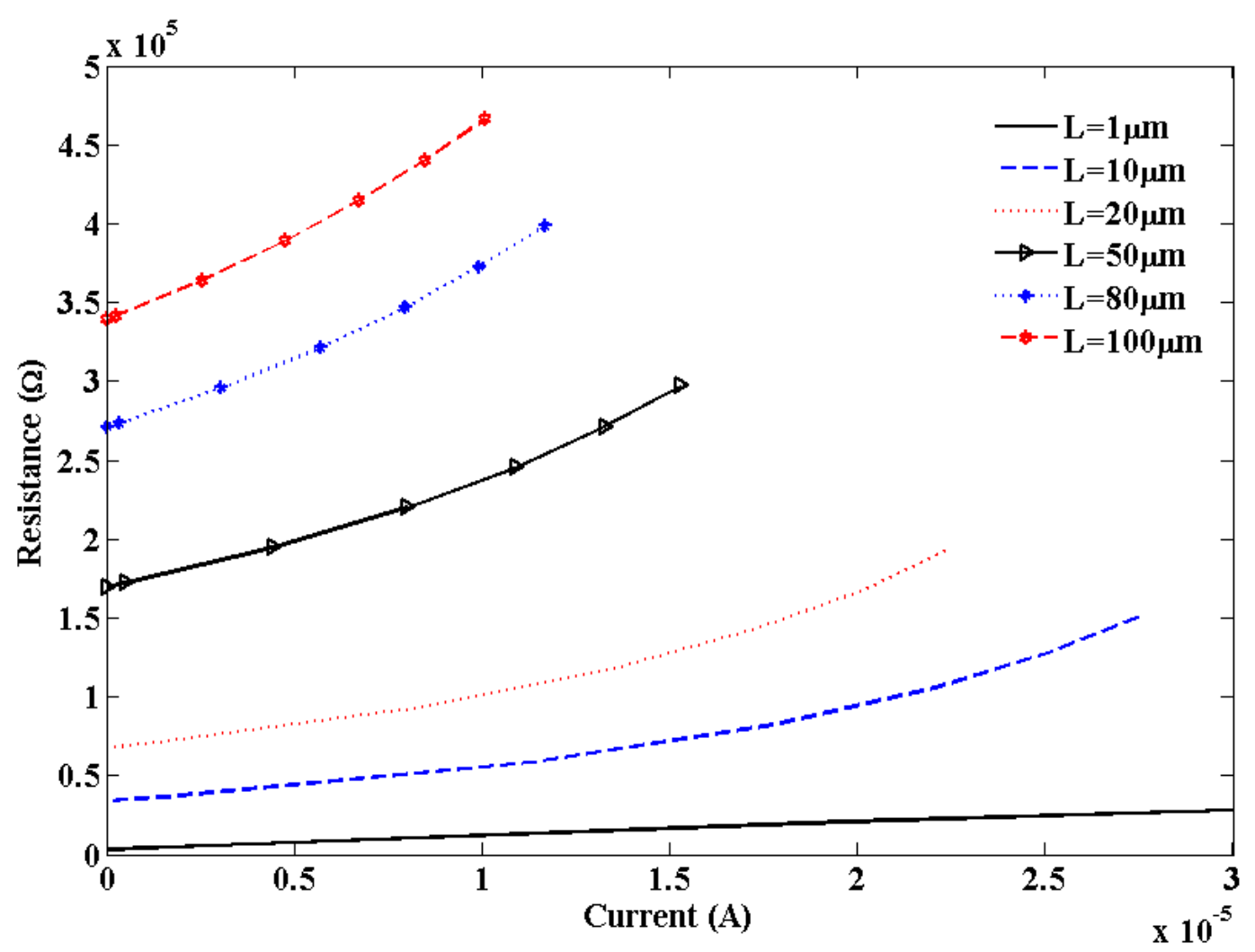

Figure 8. $\mathrm{S}_{12}$ parameter of SWCNT interconnects at different bias voltages.

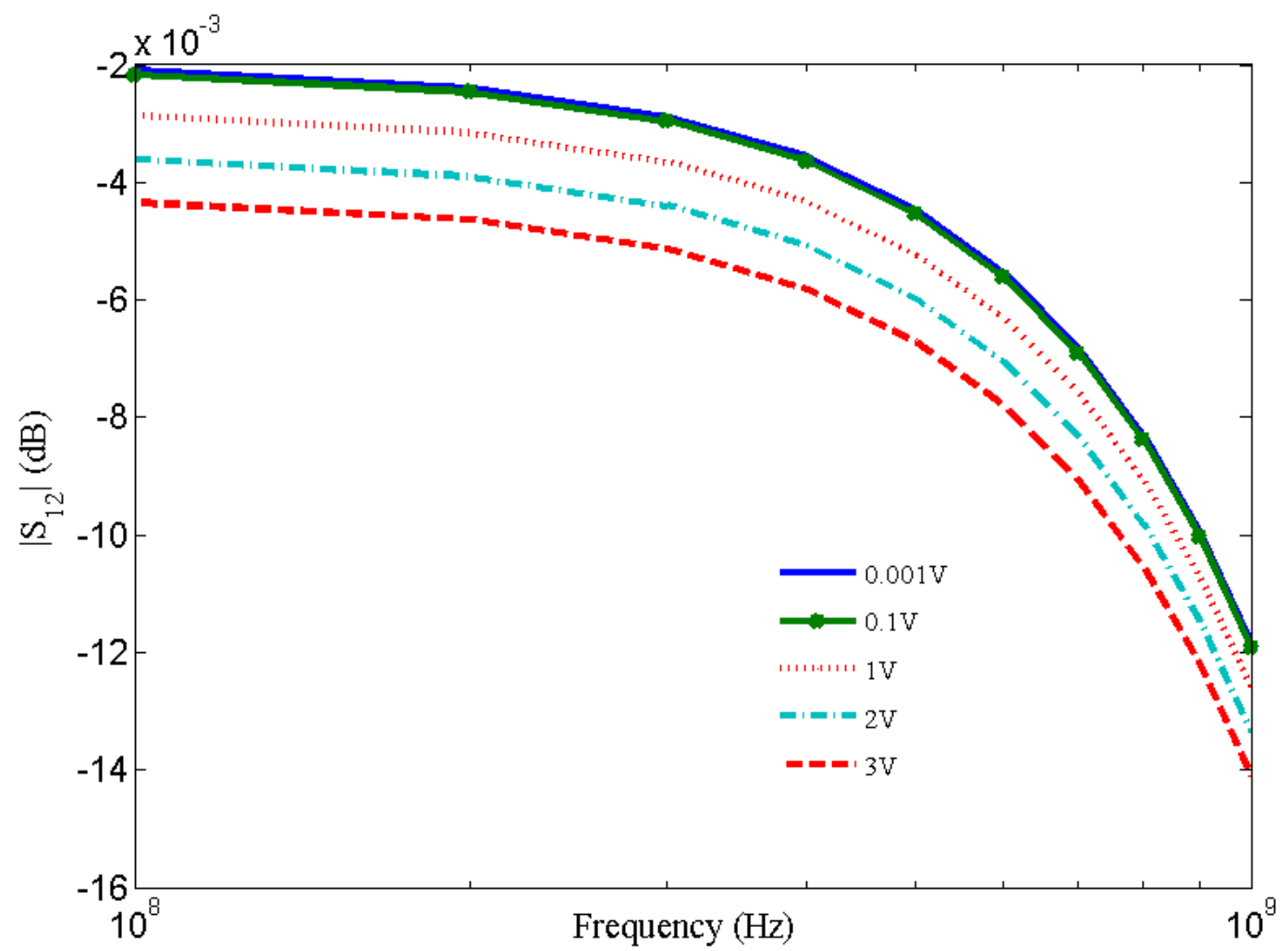




\section{Conclusions}

In this work, we have incorporated Joule heating induced phenomenon in 1D fluid model of CNT interconnects. We have studied scattering parameters of SWCNT for short, local and global interconnect lengths with different biasing voltages. We have observed that the bias voltage does not greatly affect the scattering parameters; on the other hand it significantly influences Joule heating. The breakdown shown in resistance versus current is clearly notable. The presented thermal model is very useful in experimental studies related to CNT integrated in nm- and sub-nm CMOS technology.

\section{Acknowledgments}

Part of the work is supported by the United States Air Force Research Laboratory under agreement number FA9453-10-1-0002. The U.S. Government is authorized to reproduce and distribute reprints for Government purposes notwithstanding any copyright notation thereon.

\section{References}

1. Ryu, C.; Kwon, K.-W.; Loke, A.L.S.; Lee, H.; Nogami, T.; Dubin, V.M.; Kavari, R.A.; Ray, G.W.; Wong, S.S. Microstructure and reliability of copper interconnects. IEEE Trans. Electron. Dev. 1999, 46, 1113-1120.

2. Koo, K.-H.; Kapur, P.; Saraswat, K.C. Compact performance models and comparison for gigascale on-chip global interconnect technologies. IEEE Trans. Electron. Dev. 2009, 56, 1787-1798.

3. Li, H.; Xu, C.; Srivastava, N.; Banerjee, K. Carbon nanomaterials for next-generation interconnects and passives: Physics, status, and prospects. IEEE Trans. Electron. Dev. 2009, 56, 1799-1821.

4. Iijima, S. Helical microtubules of graphitic carbon. Nature 1991, 354, 56-58.

5. Haselman, M.; Hauck, S. The future of integrated circuits: A survey of nanoelectronics. Proc. IEEE 2010, 98, 11-38.

6. Maffucci, A. Carbon nanotubes in nanopackaging applications. IEEE Nanotechnol. Mag. 2009, 3, $22-25$.

7. Chiariello, A.G.; Miano, G.; Maffucci, A. Carbon Nanotube Bundles as Nanoscale Chip to Package Interconnects. In Proceedings of 9th IEEE Conference on Nanotechnology (IEEE-NANO 2009), Genoa, Italy, 26-30 July 2009; pp. 58-61.

8. Ting, J.-H.; Chiu, C.-C.; Huang, F.-Y. Carbon nanotube array vias for interconnect applications. J. Vac. Sci. Technol. B, 2009, 27, 1086-1092.

9. Alam, N.; Kureshi, A.K.; Hasan, M.; Arslan, T. Carbon Nanotube Interconnects for Low-Power High-Speed Applications. In Proceedings of IEEE International Symposium on Circuits and Systems (ISCAS 2009), Taipei, Taiwan, 24-27 May 2009; pp. 2273-2276.

10. Stan, M.R.D.; Ghosh, A.; Tseng, F. Graphene Devices, Interconnect and Circuits. In Proceedings of IEEE International Symposium on Circuits and Systems (ISCAS 2009), Taipei, Taiwan, 24-27 May 2009; pp. 69-72. 
11. Santini, C.A.; Volodin, A.; Haesendonck, C.V.; Gendt, S.D.; Groeseneken, G.; Vereecken, P.M. Carbon nanotube-carbon nanotube contacts as an alternative towards low resistance horizontal interconnects. Carbon 2011, 49, 4004-4012.

12. Santini, C.A.; Vereecken, P.M.; Haesendonck, C.V. Growth of carbon nanotube branches by electrochemical decoration of carbon nanotubes. Mater. Lett. 2012, 88, 33-35.

13. Burke, P.J. Lüttinger Liquid Theory as a model of the gigahertz electrical properties of carbon nanotubes. IEEE Trans. Nanotechnol. 2002, 1, 129-144.

14. Salahuddin, S.; Lundstrom, M.; Datta, S. Transport effects on signal propagation in quantum wires. IEEE Trans. Electron Devices 2005, 52, 1734-1742.

15. Maffucci, A.; Miano, G.; Villone, F. A transmission line model for metallic carbon nanotube interconnects. Int. J. Circuit Theory Appl. 2008, 36, 31-51.

16. Xu, Y.; Srivastava, A. A model for carbon nanotube interconnects. Int. J. Circ. Theor. Appl. 2010, 38, 559-575.

17. Srivastava, A.; Xu, Y.; Sharma, A.K. Carbon nanotubes for next generation very large scale integration interconnects. J. Nanophotonics. 2010, 4, 1-26.

18. Pop, E.; Mann, D.; Reifenberg, J.; Goodson, K.; Dai, H. Electro-Thermal Transport in Metallic Single-Wall Carbon Nanotubes for Interconnect Applications. In Proceedings of Electron Devices Meeting, Washington, DC, USA, 5 December 2005; doi:10.1109/IEDM.2005.1609321.

19. Pop, E.; Mann, D.A.; Goodson, K.E.; Dai, H. Electrical and thermal transport in metallic single-wall carbon nanotubes on insulating substrates. J. Appl. Phys. 2007, 101, doi:10.1063/1.2717855.

20. Naeemi, A.; Meindl, J.D. Impact of electron-phonon scattering on the performance of carbon nanotube interconnects for GSI. IEEE Electron Device Lett. 2005, 26, 476-478.

21. Naeemi, A.; Meindl, J.D. Physical modeling of temperature coefficient of resistance for singleand multi-wall carbon nanotube interconnects. IEEE Electron Device Lett. 2007, 28, 135-138.

22. Huang, N.Y.; She, J.C.; Chen, J.; Deng, S.Z.; Xu, N.S.; Bishop, H.; Huq, S.E.; Wang, L.; Zhong, D.Y.; Wang, E.G.; et al. Mechanism responsible for initiating carbon nanotube vacuum breakdown. Phys. Rev. Lett. 2004, 93, 075501.

23. Santini, C. From Surface Plasmon Resonance Based Sensors to Carbon Nanotube Based Sensors. Ph.D. Thesis, Katholieke Universiteit, Leuven, Belgium, April 2011.

24. Kitsuki, H.; Yamada, T.; Fabris, D.; Jameson, J.R.; Wilhite, P.; Suzuki, M.; Yang, C.Y. Length dependence of current-induced breakdown in carbon nanofiber interconnects. Appl. Phys. Lett. 2008, 92, 173110.

25. Yamada, T.; Saito, T.; Fabris, D.; Yang, C.Y. Electrothermal analysis of breakdown in carbon nanofiber interconnects. IEEE Electron Device Lett. 2009, 30, 469-471.

26. Roche, S.; Jiang, J.; Torres, L.; Saito, R. Charge transport in carbon nanotubes: Quantum effects of electron-phonon coupling. J. Phys. Condens. Matter 2007, 19, 183203-183223.

27. Slepyan, G.Y.; Maksimenko, S.A.; Lakhtakia, A.; Yevtushenko, O.; Gusakov, A.V. Electrodynamics of carbon nanotubes: Dynamic conductivity, impedance boundary conditions, and surface wave propagation. Phys. Rev. B 1999, 60, 17136-17149.

28. Carslaw, H.S.; Jaeger, J.C. Conduction of Heat in Solids; Oxford University Press: Oxford, UK, 1959. 
29. Yamada, T.; Madriz, F.R.; Yang, C.Y. Inductance in one dimensional nanostructures. IEEE Trans. Electron Devices 2009, 56, 1834-1839.

30. Van der Veen, M; Vereecke, B. Interuniversity Microelectronics Centre (IMEC): Leuven, Belgium. Personal communication, 2011.

(C) 2013 by the authors; licensee MDPI, Basel, Switzerland. This article is an open access article distributed under the terms and conditions of the Creative Commons Attribution license (http://creativecommons.org/licenses/by/3.0/). 